\title{
Exotics and BSM in ATLAS and CMS (Non dark matter searches)
}

\author{
Li Yuan* ${ }^{*}$ \\ Beihang University, China \\ E-mail: li.yuanecern.ch
}

The Large Hadron Collider at CERN finished the Run 2 data taking by the end of 2018. Both the ATLAS and CMS experiments have recorded about $140 \mathrm{fb}^{-1}$ of proton-proton collision data at $\sqrt{s}=13 \mathrm{TeV}$ during this period. Such an enormous amount of collision data provide good opportunities for new physics searches Beyond Standard Model (BSM). New phenomena except mainstream supersymmetry are usually referred to as exotica. In this talk, several analyses of exotica searches, based on Run 2 data and completed by the ATLAS and CMS collaborations by summer 2019 are presented. Analyses directly related to dark matter searches are covered by other dedicated talks. No significant excess above the Standard Model prediction is observed in the results shown in this contribution. More stringent limits have been set on the related models.

Corfu Summer Institute 2019 "School and Workshops on Elementary Particle Physics and Gravity" (CORFU2019)

31 August - 25 September 2019

Corfù, Greece

\footnotetext{
*Speaker.

${ }^{\dagger}$ Talk on behalf of the ATLAS and CMS collaborations.
} 


\section{Introduction}

The Standard Model (SM) of particle physics has proven to be rather successful. In July 2012, the last elementary particle (the Higgs boson, a.k.a the Brout-Englert-Higgs boson) predicted by the SM was discovered by the ATLAS [1] and CMS [2] experiments at the Large Hadron Collider (LHC). However, there are still several questions which cannot be explained by the SM, for instance:

- why do neutrinos have mass ?

- why are matter and anti-matter asymmetric ?

- what is dark matter?

There is a tremendous number of models that have been introduced, aiming to extend the SM and answer the questions mentioned above. In these models, phenomena such as new particles or new physics processes would be predicted. Usually, new phenomena except mainstream supersymmetry are referred to as exotica. Search for exotica is one of main goals for LHC experiments.

During 2015 - 2018, the LHC successfully delivered proton-proton (p-p) collision data at $\sqrt{s}=13 \mathrm{TeV}$. The ATLAS and CMS experiments have recorded in total about $140 \mathrm{fb}^{-1}$ of data which are good for physics analysis [3, 4], as shown in Fig 1. Such a large amount of collision data provides good opportunities for the search for new phenomena.
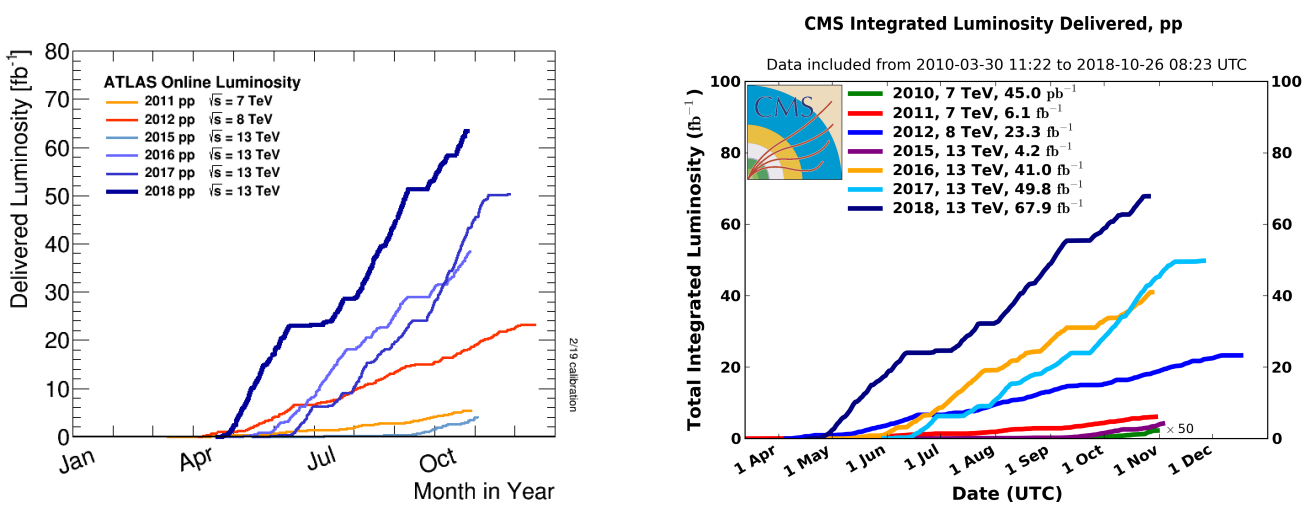

Figure 1: Luminosity recorded by the ATLAS experiment [3] (left) and the CMS experiment [4] (right) in each year of data taking during 2010-2018.

In this contribution, several representative analyses conducted by the ATLAS and CMS experiments for exotica searches are presented, including new boson searches, vector like quark searches and long lived particle searches. Given the limited space, it is impossible to cover all the analyses related to exotica searches. Only parts of the results published during 2018-2019 are shown for the selected topics. The analyses directly related to dark matter (DM) searches are not included in this talk since they are covered in other dedicated talks.

\section{New boson searches}

Numerous models predict the existence of a new boson, such as the extension of the SM in Grand Unified Theories (GUTs) like superstring, the Sequential Standard Model (SSM), the 
Heavy Vector Triplet model (HVT) and the Randall-Sundrum model (RS) with a warped extra dimension. The new boson can decay to a pair of SM particles, such as leptons, light quarks (including $u, d, s, c, b)$, photons or intermediate particles like $W / Z$ bosons and top quarks. In the experiment, one could hunt for the new resonances by looking for a bump in the reconstructed invariant mass distribution built from the decayed products.

\subsection{Dilepton}

Generally speaking, all the new particles that can decay to pairs of electrons or muons are called $Z^{\prime}$. The typical benchmark models include the Sequential Standard Model $Z_{\mathrm{SSM}}^{\prime}$ which has the same fermion couplings as the $Z$ boson in $\mathrm{SM}$ and the $Z_{\psi}^{\prime}$ in the $\mathrm{E}_{6}$-motivated Grand Unification model. Either $Z_{\mathrm{SSM}}^{\prime}$ or $Z_{\psi}^{\prime}$ is a singlet with spin-1, thus they are assumed to have no coupling with $W / Z$ bosons in SM. The dilepton channel is considered as a golden channel in the search for $Z^{\prime}$, given its simple event signature and excellent energy resolution in the lepton reconstruction. By the summer of 2019, inclusive searches for $Z^{\prime}$ with the full Run 2 data were already performed by both ATLAS [5] and CMS [6] experiments. In both analyses, functional forms for signal and background processes are summed to fit to the dilepton invariant mass distribution in real data to determine the background contribution. No significant deviation from the background expectation is observed. Limits are set on the fiducial cross section of $Z^{\prime} \rightarrow l l$ in the ATLAS experiment for the two benchmark models $Z_{\mathrm{SSM}}^{\prime}$ and $Z_{\psi}^{\prime}$, as shown in the top two plots in Fig 2. In the CMS experiment, limits are set on the ratio of the fiducial cross section of $Z^{\prime} \rightarrow l l$ in the high mass
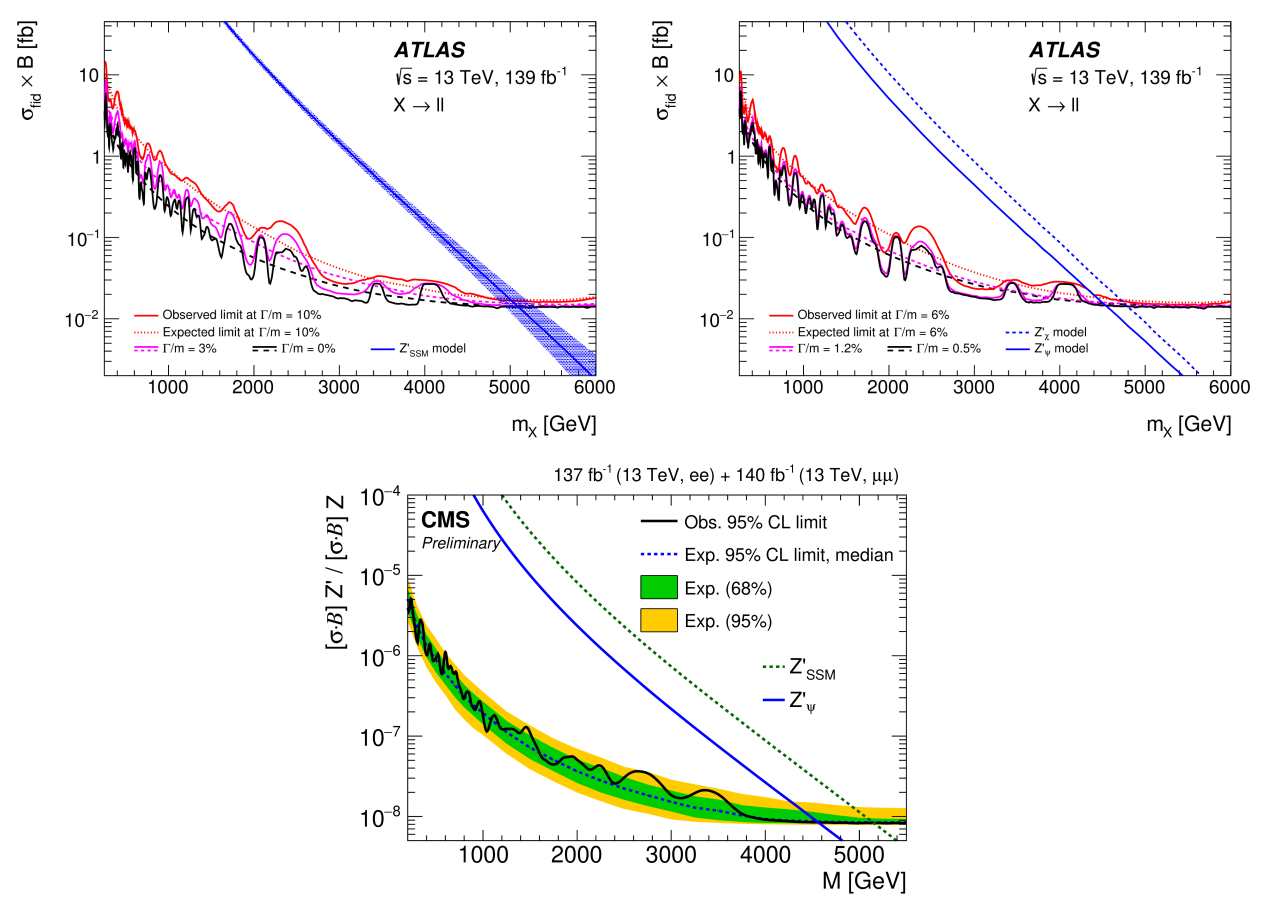

Figure 2: The upper limits at 95\% CL on the cross section of $Z^{\prime} \rightarrow l l$ as a function of $Z^{\prime}$ mass in the ATLAS experiment [5] for $Z_{\mathrm{SSM}}^{\prime}$ (top left) and $Z_{\psi}^{\prime}$ (top right). The bottom plot shows the upper limits at $95 \%$ $\mathrm{CL}$ on the ratio of the cross sections of $Z^{\prime} \rightarrow l l$ and $Z \rightarrow l l$ in SM as a function of $Z^{\prime}$ mass in the CMS experiment [6]. 
region and the fiducial cross section of $Z \rightarrow l l$ in the $Z$ mass peak, as shown in the bottom plot in Fig 2, which benefits from the cancellation of the systematic uncertainties that are independent of mass. As the results from the ATLAS and CMS experiments show, $Z_{\mathrm{SSM}}^{\prime}$ with masses lower than $5 \mathrm{TeV}$ and $Z_{\psi}^{\prime}$ with masses lower than $4.5 \mathrm{TeV}$ are excluded at 95\% CL.

In the CMS experiment, a search for a narrow resonance decaying into a pair of muons in the low mass range (i.e $11.5-200 \mathrm{GeV}$ ) is also performed by using the full Run 2 data [7]. The mass range of $75-110 \mathrm{GeV}$ is omitted since it is dominated by the $Z$ boson. In order to probe the very low di-muon mass region, a special trigger technique which is called data scounting is adopted to select and record events. In this technique, a small set of significantly lower muon $p_{\mathrm{T}}$ threshold triggers is implemented and a very limited amount of event information is recorded. The total integrated luminosity of these scounting data in Run 2 is $\sim 100 \mathrm{fb}^{-1}$. The di-muon resonance search in the mass range of $11.5-45 \mathrm{GeV}$ is performed using these scounting data, while the search in $45-75$ and $110-200 \mathrm{GeV}$ mass range is performed using the fully reconstructed data. The results are interpreted in the context of the dark photon model $Z_{\mathrm{D}}$. Given the fact that the data show no significant deviation from SM predictions, upper limits at $90 \% \mathrm{CL}$ are set on $\varepsilon^{2}$ (where $\varepsilon$ is the kinetic mixing coefficient between $Z_{\mathrm{D}}$ and SM fermions) as a function of the $Z_{\mathrm{D}}$ mass, as shown in Fig 3.

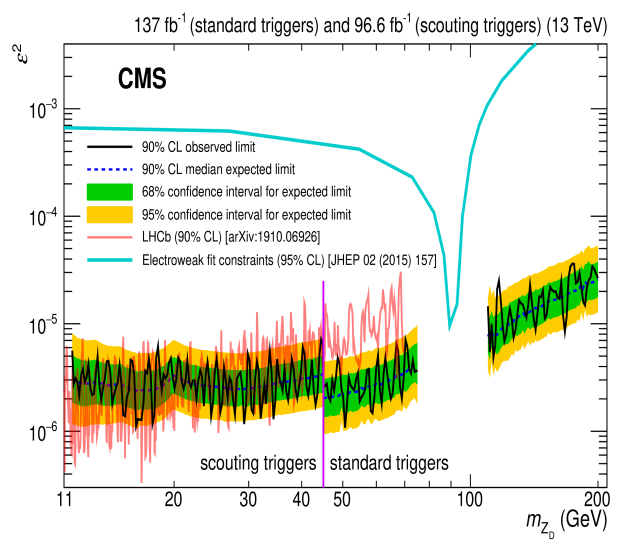

Figure 3: The upper limits at $90 \% \mathrm{CL}$ on $\varepsilon^{2}$ as a function of the $Z_{\mathrm{D}}$ mass in the CMS experiment [7]. The limits at $95 \%$ CL obtained through the electroweak constraints are shown in light blue [8], and the results obtained from the $\mathrm{LHCb}$ experiment are shown in red [9].

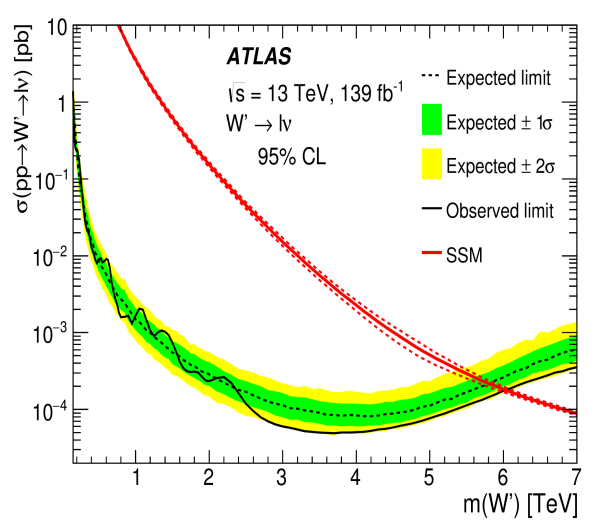

Figure 4: The upper limits at $95 \% \mathrm{CL}$ on the cross section of $W^{\prime} \rightarrow l v$ as a function of the $W^{\prime}$ mass in the SSM after combining electron and muon channels in the ATLAS experiment [10].

\subsection{Single lepton}

A search for a heavy charged boson decaying to an energetic electron or muon and a neutrino is performed by the ATLAS experiment using the full Run 2 data [10]. The interpretation in this analysis is done with the benchmark model (SSM) in which a heavy spin-1 boson $W^{\prime}$ is predicted. The $W_{\mathrm{SSM}}^{\prime}$ is assumed to have the same coupling strength to fermions as the $W$ boson in the SM but with no coupling to $W / Z$ bosons in SM. Due to the presence of an undetected neutrino in the final state, it is impossible to fully reconstruct the invariant mass of the lepton and neutrino. Instead, 
the transverse mass $\left(m_{\mathrm{T}}\right)$ constructed from lepton transverse momentum and missing transverse momentum is used as the discriminating variable between signal and background events. The $m_{\mathrm{T}}$ distribution is then divided into dozens of bins, and each bin is considered as a counting experiment. After combining all the bins with the likelihood which also includes the uncertainties implemented as log-normal constraints, the fit is then performed. Given that no significant excess above the SM expectation is observed, upper limits are set on the cross section of $W^{\prime} \rightarrow l v$, as shown in Fig 4. A $W_{\mathrm{SSM}}^{\prime}$ with mass below $6 \mathrm{TeV}$ is excluded at $95 \% \mathrm{CL}$.

\subsection{Dijet}

The dijet channel is one of the most promising channels to probe resonances in the extremely high mass region. Both the ATLAS [11] and CMS [12] experiments have conducted searches for dijet resonances in wide range of the $m_{j j}$ spectrum using the full Run 2 data. The results are interpreted in several new physics scenarios, including excited quarks from compositeness models, heavy $W^{\prime}$ and $Z^{\prime}$ bosons, a chiral excitation of the $W$ boson, quantum black holes and KaluzaKlein gravitons. Since no sign is found for new resonances, limits are set on the cross section as a function of the resonance mass.

Based on the Run 2 data, it is actually a big challenge to probe the low mass regime especially the mass range below $200 \mathrm{GeV}$. This is because the background multijet events increase significantly, and higher jet trigger thresholds need to be applied in order to control the online event rates as the collision energy and beam intensity increased in Run 2. One effective approach to explore the low mass regime is to require an energetic ISR jet or ISR photon to trigger the events. In those events, the resonance is highly boosted so that the decay products are merged into a single wide jet. In this case, jet substructure techniques are crucial to discriminate the dijet candidates from the single jet candidates. There are several analyses adopting this approach that have been completed by the ATLAS and CMS experiments. One analysis performed by the CMS experiment [13] has probed the dijet resonance down to the mass region of $10-50 \mathrm{GeV}$. This is the first analysis from LHC experiments in the search for dijet resonances in such a low mass region.

There is no significant excess above the SM prediction observed in any analysis for the dijet resonance search. A comprehensive summary of the results related to the $Z^{\prime}$ search in the dijet channel obtained by the summer of 2019 is shown in Fig 5 .

\subsection{Diboson}

Resonances of dibosons are predicted in several extensions of the SM. A search for narrow diboson resonances (including $W Z / Z Z / W W$ ) decaying to fully hadronic final states with full Run 2 data is performed by the ATLAS experiment [15]. In this analysis, given the highly boosted topology of the vector bosons, the decayed products from the vector boson are then merged together forming a single wide jet. The final signature of such diboson events becomes two wide jets. In order to greatly suppress the huge background contribution from the multijet events, dedicated jet substructure techniques (i.e $W / Z$ tagging) need to be applied. There are several improvements with respect to the previous analyses, such as including the track information in the calorimeter based jet reconstruction, and including the jet mass and other more jet substructure variables in the boosted boson finding. An example of the dijet invariant mass distributions in this analysis is shown in 


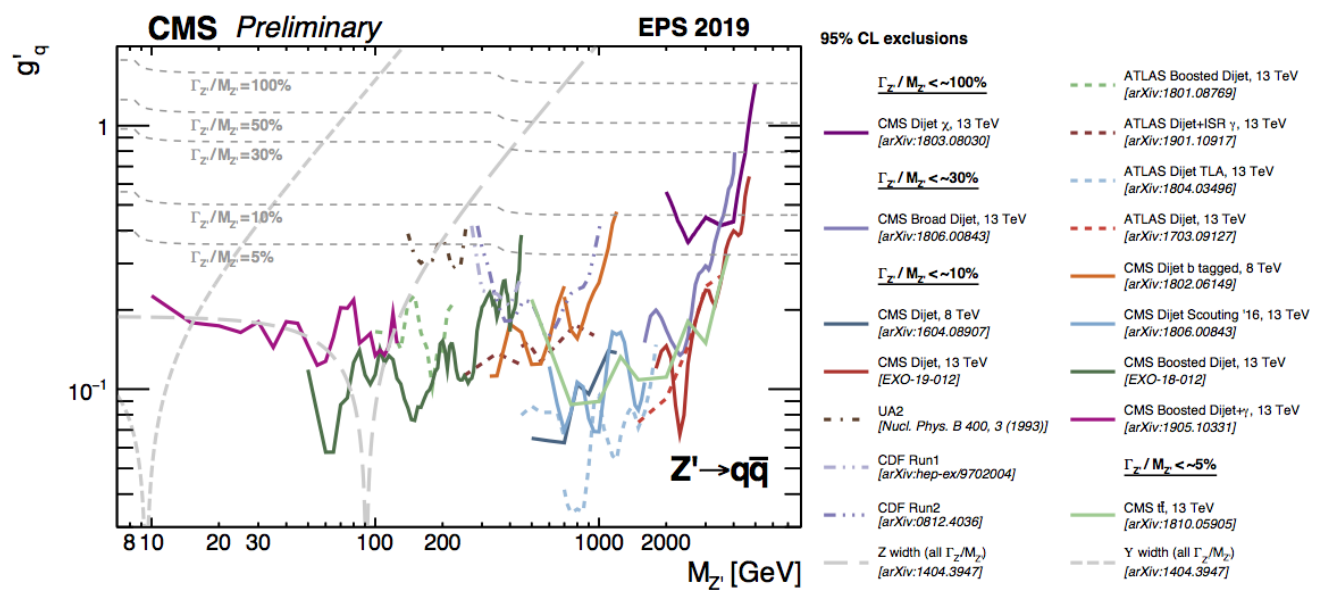

Figure 5: Limits on the universal coupling $\left(g_{q}^{\prime}\right)$ between a $Z^{\prime}$ boson and quarks as a function of $Z^{\prime}$ boson mass [14]. All the related results performed by the ATLAS, CMS, CDF and UA2 experiments by the summer of 2019 are included.

Fig 6. A similar analysis is also performed by the CMS experiment [16] using 2016 and 2017 data. In this analysis, a novel method is applied to estimate the background, i.e. performing a 3dimensional maximum likelihood fit on the two jets masses and the dijet invariant mass. With such a novel method, the sensitivity is improved by $\sim 30 \%$. An example dijet invariant mass distribution from this analysis is also shown in Fig 7. In both of the analyses, no significant deviation from the background expectation is observed. Limits are set on the cross section of $X \rightarrow V V$ as a function of the resonance mass.

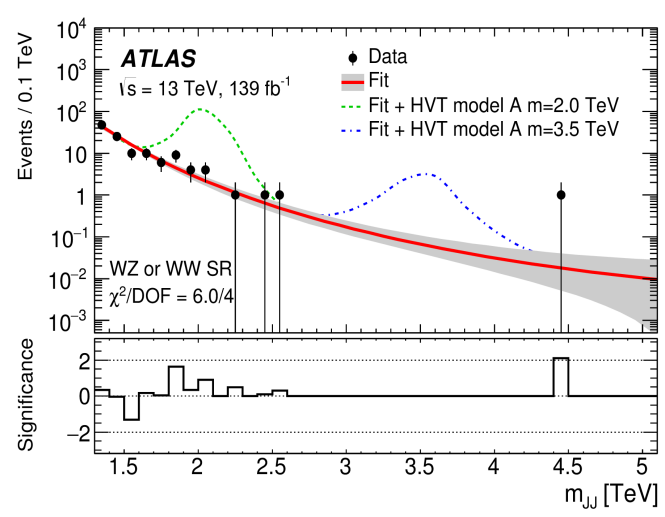

Figure 6: The dijet invariant mass distribution in data after tagging with $W W+W Z$ in the analysis of the diboson resonance search from the ATLAS experiment [15]. The lower panel shows the significance.



Figure 7: The dijet invariant mass distribution for the events with a jet mass in the region of [55, 215] $\mathrm{GeV}$ in the diboson resonance search in the CMS experiment [16]. The lower pannel shows the ratio of data events and SM background expectation. 


\section{Vector-Like Quarks}

Vector-Like Quarks (VLQs) are an extra family of spin- $\frac{1}{2}$ quarks, which are predicted by many new physics models, such as the composite Higgs model and the "little-Higgs" model. They do not obtain their masses through the Yukawa coupling to the Higgs boson, thus their existence will not affect the Higgs boson production and decay and help to resolve the hierarchy problem. The VLQs with electric charge of $+\frac{2}{3} e$ and $-\frac{1}{3} e$ are labelled as $T$ and $B$ respectively. As for the top quark, single production and pair production of VLQs are possible. Single production is through the electroweak interaction and dominates in the high VLQ mass region $(\sim 2 \mathrm{TeV})$. The pair production is from strong interaction and with a rate comparable with single production in the mass region around $1 \mathrm{TeV}$. The VLQs are expected to have symmetric vector-like couplings to $W / Z$ bosons, and their decay modes include:

- $T \rightarrow b W, T \rightarrow t Z, T \rightarrow t H$,

- $B \rightarrow t W, B \rightarrow b Z, B \rightarrow b H$.

In a publication by the CMS collaboration [17], two independent analyses about searching for VLQs in fully hadronic final states are presented. The first analysis targets the pair production of $T$ in the channel of $T T \rightarrow b W b W$. In this analysis, the traditional cut-based strategy is adopted, by applying the dedicated $W$-tagging and $b$-tagging algorithms to reconstruct the VLQ $T$ quarks in the events. At least four jets are required to be present in each event, and the scalar sum of the jet transverse momenta $\left(H_{\mathrm{T}}\right)$ is used as the discriminating variable. The second analysis targets all the decay modes of $T$ and $B$, by applying Neural Network algorithms to identify the jets to be consistent with one of the six highly boosted particles: top, $W$ boson, $Z$ boson, Higgs boson, $b$ quark and a light quark or gluon. The numbers of jets identified as one of the six boosted particles are then used to define 126 different signal regions. $H_{T}$ is also used as a discrimination variable in this complicated analysis. In both analyses, the data show consistency with the SM expectation, thus limits are set for the ratio of the production cross section and the predicted cross section, as shown in Fig 8. The VLQ with electric charge of $-\frac{4}{3} e$ is also allowed in many models, which is usually labelled as $Y$. In an analysis by the ATLAS experiment [18], the search for single production of a VLQ $T$ or $Y$ is performed using the 2016 data. Due to the charge of $Y$, it can only decay into $b W$. VLQ $T$ is assumed to only decay into $b W$ in this analysis. The leptonic decay channel of the $W$ boson is used. In order to estimate the dominate backgrounds, two control regions are defined. They are also being fitted in the final binned likelihood fit to normalize the enriched particular background sources and constrain the uncertainties. As shown in the left plot of Fig 9, good agreement is found between data and background expectation in the search for the $Y$ quark. Thus, limits are set at $95 \% \mathrm{CL}$ on the cross section of the $Y$ quark as a function of its mass, as shown in the right plot of Fig 9.

\section{Long-Lived Particles}

BSM particles may have lifetimes that are long compared to SM particles. Such particles are referred to as Long-Lived Particles (LLP), which would decay far from the interaction point. 

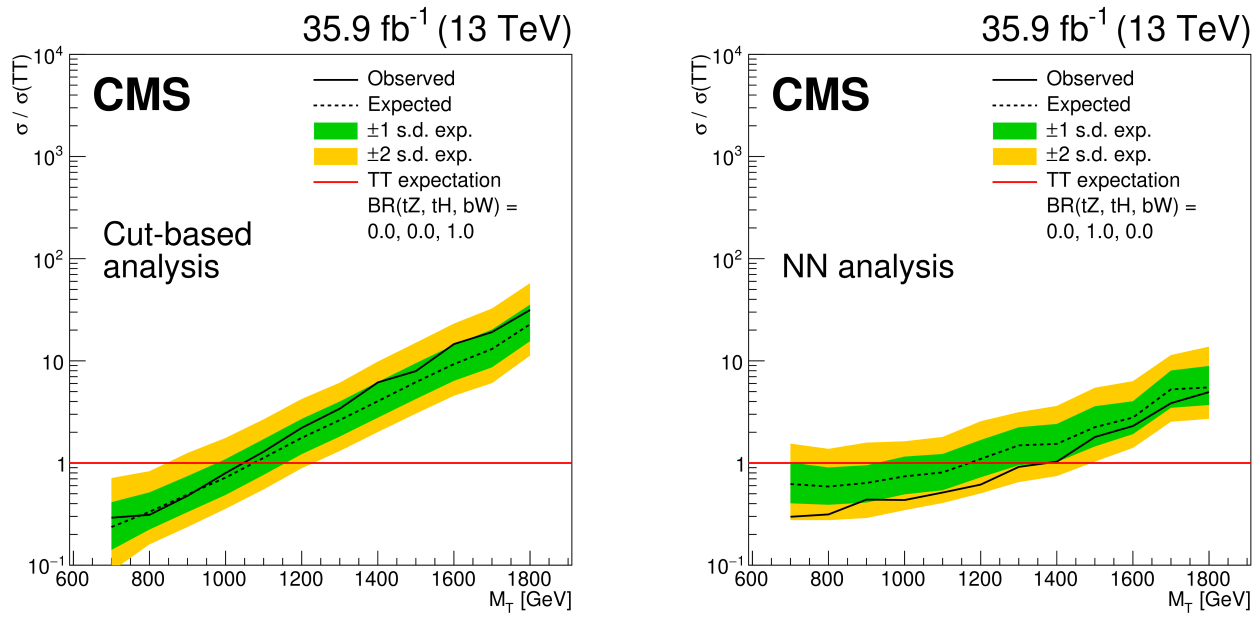

Figure 8: Limits at $95 \% \mathrm{CL}$ on the ratio of the production cross section and the predicted cross section of pair production of $T$ in the CMS experiment [17]. The left plot shows the results from the cut-based analysis where the branching fraction of $T \rightarrow b W$ is assummed to be 1 . The right plot shows the results from a neural network analysis where the branching fraction of $T \rightarrow t H$ is assummed to be 1 .
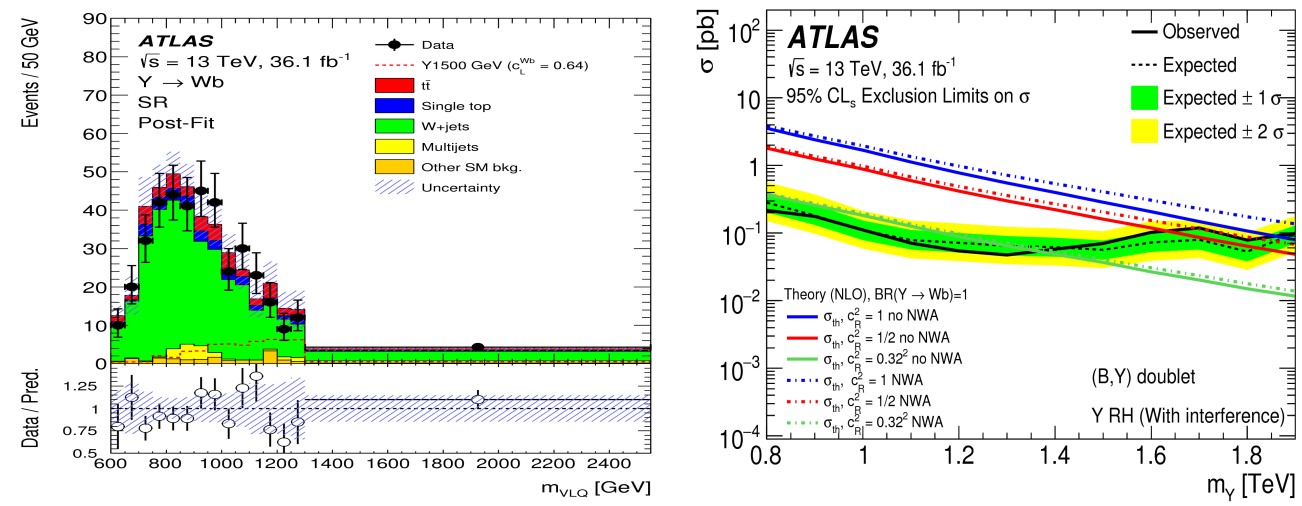

Figure 9: The left plot shows the distribution of the VLQ candidate mass after the final selection in the search for single production of the $Y$ quark performed by the ATLAS experiment [18]. The lower panel shows the ratio of data to the fitted background yields. The right plot shows the limits at 95\% CL on the cross section of the $Y$ quark as a function of its mass.

The LLP show up in detectors in different signatures, such as displaced leptons or displaced jets, delayed photons, emerging jets, disappearing or kinked tracks and so on. As a consequence, the search for LLP requires customised techniques to identify displaced vertices. The LHC detectors were not originally designed to detect LLP, however, it is still feasible for LHC experiments to conduct searches for LLP. In fact, lots of related analyses have already been performed by the LHC experiments, and the program for LLP searches is rapidly expanding. In this contribution, three analyses performed by the ATLAS and CMS experiments about the LLP searches are presented.

A search for LLP with the final signature of displaced jets and missing transverse momentum is performed by the CMS experiment using the full Run 2 data [19]. The models which can predict 
such signatures include supersymmetry (SUSY) with gauge-mediated SUSY breaking (GMSB), split and stealth SUSY and hidden valley models. The ECAL timing information is for the first time applied to searches for the displaced jets, which helps to reduce the backgrounds significantly with marginal signal efficiency loss. No deviation from the SM is observed and limits are set on the gluinos from the GMSB model. The analysis excludes at 95\% CL the GMSB gluinos with masses of 2.1, 2.5 and $1.9 \mathrm{TeV}$ for decay lengths of $0.3,1,100 \mathrm{~m}$ respectively.

Another interesting search with the signature of one or two photons with a displaced vertex and at least three jets is also performed by the CMS experiment using 2016 and 2017 data [20]. As predicted by GMSB, pair-produced squarks and gluinos (decaying into a squark and a quark) undergo cascade decays. The squark first decays into a SM quark and neutralino: $\widetilde{q} \rightarrow q \widetilde{\chi}_{1}^{0}$. Then the neutralino decays into a photon (or $Z$ boson) and a gravitino $(\widetilde{G})$. Depending on the effective scale of SUSY breaking, the coupling between the neutralino and gravitino could be very weak and lead to a long neutralino lifetime. In that case, the photon from the neutralino decay would be displaced. The initial pair production of squarks or gluinos would also introduce additional energetic jets. In order to reconstruct delayed photons, the ECAL timing information is used. On the other hand, the delayed photons tend to show more elliptical eletromagnetic showers in the $\eta-\phi$ plane, dedicated observables are defined based on this feature. In this search, neutralinos with masses of 320, 525, 360 and $215 \mathrm{GeV}$ are excluded at 95\% CL for decay lengths of $0.1,1,10$, and $100 \mathrm{~m}$ respectively.

The ATLAS experiment conducted a search for Heavy Neutral Leptons (HNL) with 2015 and 2016 data [21]. Introducing a neutrino mass-generation mechanism can help to explain why the neutrinos are massive. One example of such a mechanism is the Type-1 Seesaw mechanism [22], which introduces a right-handed Majorana neutrino (denoted as a Heavy Neutral Lepton, HNL). The HNLs are produced through $W / Z$ decays. And at the LHC, the production through $W$ decays is more favored due to trigger requirements. The HNLs can decay promptly or be long-lived depending on the mixing between HNLs and neutrinos. This search targets two different signatures to probe the prompt HNL and the displaced HNL. For the prompt signature, two channels $\left(W^{ \pm} \rightarrow \mu^{ \pm} \mu^{ \pm} e^{\mp} v_{e}\right.$ and $W^{ \pm} \rightarrow e^{ \pm} e^{ \pm} \mu^{\mp} v_{\mu}$ ) are considered, which are lepton-number violated. For the displaced signature, four channels are included: $W^{ \pm} \rightarrow \mu^{ \pm} \mu^{\mp} e^{ \pm} v_{e}, W^{ \pm} \rightarrow \mu^{ \pm} \mu^{\mp} \mu^{ \pm} v_{\mu}$, $W^{ \pm} \rightarrow \mu^{ \pm} \mu^{ \pm} e^{\mp} v_{e}$ and $W^{ \pm} \rightarrow \mu^{ \pm} \mu^{ \pm} \mu^{\mp} v_{\mu}$. The first two channels are lepton-number conserved and the other two channels are lepton-number violated. The common feature for all the four channels is a prompt muon accompanied by a displaced vertex from two opposite sign leptons. The observed distributions in data are consistent with background expectations in both signatures. The search sets stringent limits on the coupling strengths (between HNLs and neutrinos) for a HNL mass range of $4.5-50 \mathrm{GeV}$.

A large number of searches for LLP have already been performed by the ATLAS and CMS experiments. An overview of the LLP searches conducted by the ATLAS experiment as of July 2019 is shown in Fig 10. The overview of the results obtained by the CMS experiment as of July 2019 for the LLP searches can be seen in Fig 11. As both figures show, a lot of signatures have been explored and the constraints obtained cover a wide range of LLP decay lengths. 


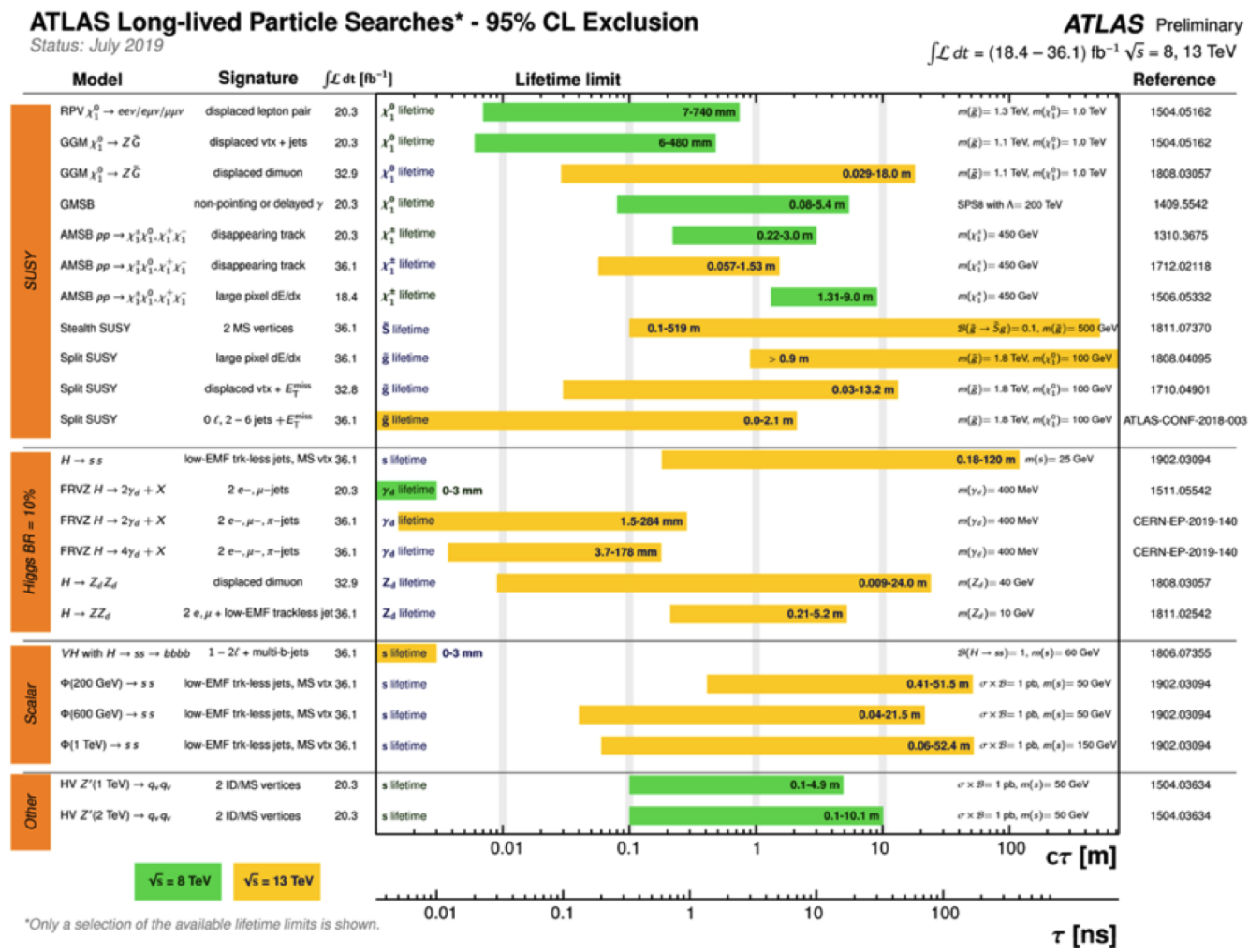

Figure 10: Overview of the results obtained in the Long-Lived Particles searches in the ATLAS experiment as of July 2019 [23].

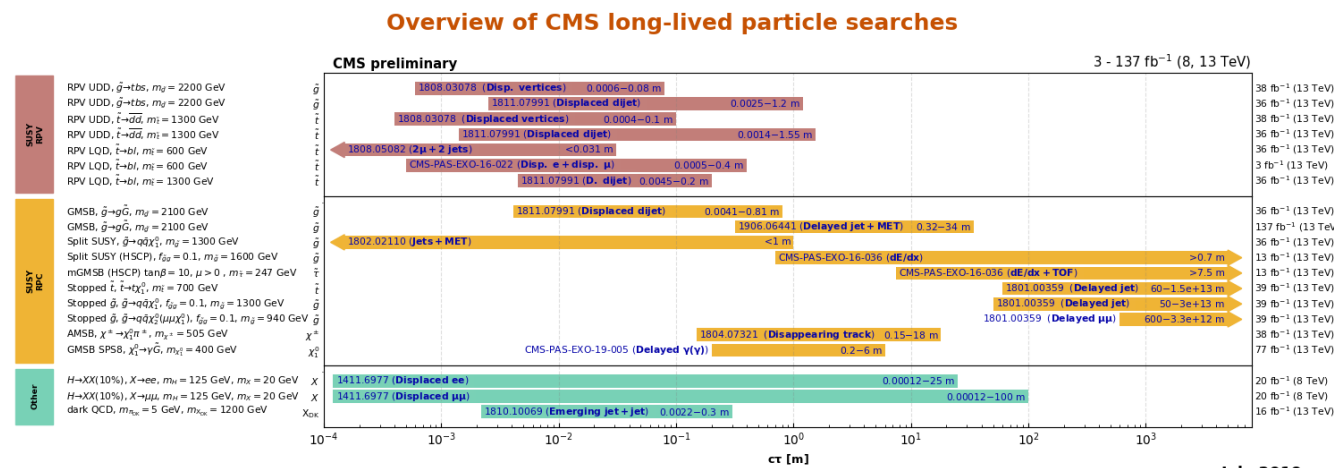

Figure 11: Overview of the results obtained in the Long-Lived Particles searches in the CMS experiment as of July 2019 [14]. 


\section{Conclusion}

The LHC ran very successfully during 2015 - 2018 for the Run 2 data taking, and delivered $\sim 140 \mathrm{fb}^{-1}$ of p-p collision data of very high quality. This large amount of data provides good opportunities for searches Beyond Standard Model, such as new bosons, vector-like quarks and long-lived particles. Both the ATLAS and CMS experiments conducted a large number of analyses with various signatures. Unfortunately no sign of new physics has been found yet. However, the new physics must reside somewhere, maybe at a higher energy scale, or maybe in some corners we have not probed yet. We need to keep exploring to find them.

\section{References}

[1] ATLAS Collaboration, JINST 3, S08003 (2008).

[2] CMS Collaboration, JINST 3, S08004 (2008).

[3] ATLAS public: https://twiki.cern.ch/twiki/bin/view/AtlasPublic/LuminosityPublicResultsRun2.

[4] CMS public: https://twiki.cern.ch/twiki/bin/view/CMSPublic/LumiPublicResults

[5] ATLAS Collaboration, Phys.Lett.B 796(2019)68

[6] CMS Collaboration, CMS-PAS-EXO-19-019

[7] CMS Collaboration, CMS-PAS-EXO-19-018

[8] D. Curtin, R. Essig, S. Gori, and J. Shelton, JHEP 02 (2015) 157

[9] LHCb Collaboration, Phys.Rev.Lett 120(2018)061801

[10] ATLAS Collaboration, Phys.Rev.D 100(2019)052013

[11] ATLAS Collaboration, ATLAS-CONF-2019-007

[12] CMS Collaboration, CMS-PAS-EXO-19-012

[13] CMS Collaboration, Phys.Rev.Lett 123(2019)231803

[14] CMS public: https://twiki.cern.ch/twiki/bin/view/CMSPublic/SummaryPlotsEXO13TeV

[15] ATLAS Collaboration, JHEP 09(2019)091

[16] CMS Collaboration, CMS-PAS-B2G-18-002

[17] CMS Collaboration, Phys.Rev.D 100(2019)072001

[18] ATLAS Collaboration, JHEP 05(2019)164

[19] CMS Collaboration, Phys.Lett.B 797(2019)134876

[20] CMS Collaboration, hys.Rev.D 100(2019)112003

[21] ATLAS Collaboration, JHEP 10(2019)265

[22] T.Yanagida, Prog.Theor.Phys 64(1980)1103

[23] ATLAS public: https://atlas.web.cern.ch/Atlas/GROUPS/PHYSICS/CombinedSummaryPlots/EXOTICS/ 